\title{
Broadcasting in Sensor Networks: The Role of Local Information
}

\author{
Sundar Subramanian, Sanjay Shakkottai and Ari Arapostathis \\ Wireless Networking and Communication Group \\ Department of ECE \\ University of Texas at Austin, Austin, TX \\ E-mail : \{ssubrama, shakkott, ari\}@ece.utexas.edu
}

\begin{abstract}
Flooding based strategies are conventionally employed to perform querying and broadcasting in sensor networks. These schemes have low hop-delays of $\Theta\left(\frac{1}{M(n)}\right)$ to reach any node that is a unit distance away, where $M(n)$ is the transmission range of any sensor node. However, in sensor networks with large radio ranges, flooding based broadcasting schemes cause many redundant transmissions leading to a broadcast storm problem. Many approaches have been proposed to mitigate the broadcast storm problem, where broadcast schemes employ some knowledge of the previous transmissions to reduce the extraneous transmissions. In this paper, we study the role of geographic information and state information (i.e. memory of previous messages or transmissions) in reducing the redundant transmissions in the network.

We consider three broadcasting schemes with varying levels of local information: (i) where nodes have no geographic or state information, (ii) nodes have coarse geographic information about the origin of the broadcast, and (iii) where nodes have no geographic information, but remember previously received messages. We also consider the related problem of broadcasting to a set of "spatially uniform" points in the network (lattice points) in the regime where all nodes have only a local sense of direction. For each of these networks, we compute the number of transmissions required to achieve broadcast delays that are order-wise equivalent to simple flooding algorithms, i.e. $\Theta\left(\frac{1}{M(n)}\right)$.

We first show that networks with no geographic or state information require exponentially large number of transmissions whereas networks with very little geographic or state information can utilize the knowledge to significantly reduce the transmission overheads. Next, we show that networks with local information, can reduce the congestion by spreading the messages more uniformly through the network. Finally, we show that networks with only state information can also employ the information to provide a radial drift to the transmitted packets. In the context of lattice broadcasting, we again show that local information results in significant reduction of transmission overheads. We quantitatively compare the transmission overheads of broadcasting strategies and validate our results using simulations.
\end{abstract}

\section{INTRODUCTION}

Advances in Micro-embedded computing systems, coupled with developments in wireless technology have enabled the mass production of small sensing devices equipped with wireless communication capabilities. It is envisaged in near future that sensors networks formed by large-scale deployment of such devices would perform distributed sensing/control operations. Applications for sensor networks include commercial applications involving macro-scale measurements and control, intrusion detection and robust communication. These

This research was partly supported by NSF Grants ACI-0305644, CNS0325788, CNS-0347400, ECS-0218207 and in part by the Office of Naval Research through the Electric Ship Research and Development Consortium.

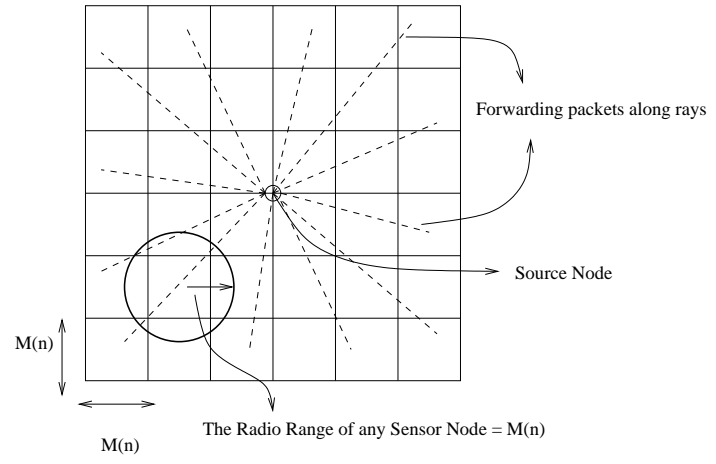

Fig. 1. Forwarding packets along straight lines - We require only one transmission per tile for broadcasting.

networks are characterized by the absence of any established architecture and by constrained energy and computational resources at each node. Communication between any two nodes in these networks is mainly through packet forwarding by intermediary relay nodes, where messages are relayed to neighbor nodes within the radio range.

In many sensor network applications, broadcasting is a common communication primitive required for various control operations. Applications regularly require broadcast operations to update global information and also to perform network maintenance such as updating topology, route discovery and propagating alarm signals. Similarly, many sensing applications periodically inform the sensors to collect information. Thus, an important communication task of a sensor network is to disseminate messages/instructions information to most nodes. A related broadcasting problem arises when a node (say, a controller or a fusion center) needs to query/send a control message to a subset of nodes which are approximately spatially uniform. Such a scenario can arise for instance when the controller needs a spatially uniform sample of a physical underlying process.

In the presence of energy and computation constrained nodes, we require that the communication operations for both these scenarios be energy efficient, computationally simple and delay sensitive. Since the channel utilized by the sensor nodes is a wireless channel, the messages are broadcast to all nodes within the radio range of the transmitting node. Efficient broadcast strategies utilize the inherent broadcast nature of the communication channel to minimize the total number of transmissions, while guaranteeing the reception of the message 


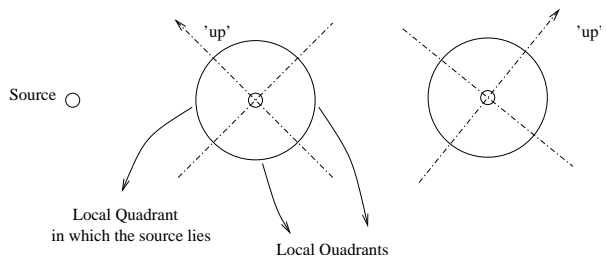

Fig. 2. Local Quadrants in Sensor nodes

at all nodes.

Broadcasting in wired networks, is conventionally performed by a simple flooding algorithm, in which each node forwards the message/query once to all its neighbors, when it first hears the message, and ignores all further receptions of the same message. Thus the nodes remember 'state' information, i.e, if a nodes has previously heard a message or not. An advantage with such a flooding based broadcast strategy is that it achieves low broadcast delays, without any geographic information at sensor nodes. However, in densely connected networks, such as sensor networks with relatively large radio ranges, such simple flooding based querying/broadcast schemes create many redundant transmissions causing energy inefficiency. Such algorithms lead to a broadcast storm [13] problem, where the same message is received at a node, multiple number of times.

If all nodes in the network had perfect geographic information, it possible to considerably reduce the total number of transmissions. Ideally one could use only $\left(\frac{1}{M(n)}\right)^{2}$ transmissions in the network, where $M(n)$ is the radio range (in other words, one transmission per tile, see Figure 1). Whereas with simple flooding, the number of transmissions would at least scale as $n$ (the number of nodes), which could be much larger in dense sensor networks.

A simple scheme to achieve a $\Theta\left(\frac{1}{M(n)}\right)^{2}$ number of transmissions ${ }^{1}$ is by dispatching packets along "rays" as shown in Figure 1. This scheme requires "perfect" geographic information at the nodes in order to make sure that the rays do not "bend" or loop back.

Similarly, if the nodes had perfect state information, where all nodes had knowledge of past transmissions and routing tables, it is possible to reduce the broadcast redundancy by constructing a minimum spanning tree or creating an overlay network. However, in many sensor networks, it is impractical to acquire perfect geographic information, as it requires sophisticated location devices and/or computational capabilities. In networks with simultaneous broadcasts by many sources, nodes are required to maintain routing information for messages from each source. Thus it is infeasible to store all routing state information, in networks with meager storage resources.

In this paper, we study the role of information (geographic and state information) on reducing the broadcast redundancy, while preserving the delay efficiency of flooding based approaches for the cases of $(i)$ broadcasting over the entire space, and (ii) broadcasting over a lattice. In particular, we consider efficient broadcast strategies in networks with varying levels of information at the nodes:

\footnotetext{
${ }^{1}$ By $g(n)=O(f(n))$, we note that there exists a positive constant $c_{1}$ such that for $n>N, g(n) \leq c_{1} f(n)$. We say $g(n)=\Omega(f(n))$, if $f(n)=$ $O(g(n)) . g(n)=\Theta(f(n))$ if $g(n)=O(f(n))$ and $f(n)=O(g(n))$.
}

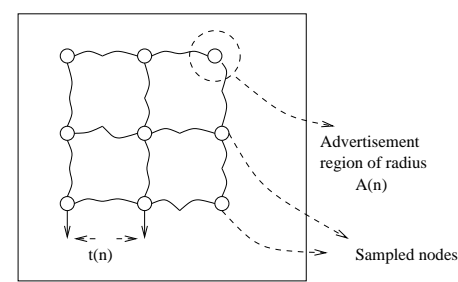

Fig. 3. Spatial sampling in Sensor nodes

1) Zero information: Nodes have no geographic information or memory, and broadcasting is only through random packet forwarding.

2) Source Quadrant Information: Nodes have a local notion of four directions, not common to all nodes, and know the local quadrant in which the source is located (See Figure 2).

3) Transmission state Information: Nodes have no geographic information, but all nodes remember messages received previously. (i.e. state information)

4) Local Direction Information: Finally, for the problem of spatial sampling (see Figure 3), we consider that nodes have an approximate sense of 'East', 'West', 'North' and 'South', but have no other geographic information, source or receiver location information or memory.

\section{A. Main Contributions}

We consider dense sensor networks on a plane, where each node has a large number of neighbor nodes, within its radio range $M(n)^{2}$. We model this by a continuum of sensor nodes, where we associate a sensor node to every point in the plane. We measure broadcast delay in terms of the number of hops required to reach any given point on the network, ignoring the queuing delay at the nodes (for a similar model, see [20],[10]). Under this network model, we quantitatively analyze the efficiency of broadcast strategies with varying levels of information at the sensor nodes.

We first observe that flooding-based strategies lead to broadcast delays that are of the same order as optimal straightline broadcasting (although with many more transmissions), i.e., the broadcast delay $D(n)=\Theta\left(\frac{1}{M(n)}\right)$. Thus, in order to compare broadcasting in networks with varying levels of information, we restrict the strategies to possess a broadcast delay $D(n)=\Theta\left(\frac{1}{M(n)}\right)$, i.e., order-wise equivalent to flooding based strategies. For a broadcast to reach a node, it is necessary for a transmission to occur within the radio range $M(n)$ of the sensor node. However, if all the neighbor nodes within a radius $A(n)$ contain routing information to direct the transmission to the intended node, the transmissions are only required to reach a ball of radius $A(n)$ about the node. Thus, it is possible for nodes to advertise the location to a radius $A(n)$, at the cost of increased local routing information about the receiving node.

We measure delay in terms of hop-count, and energy efficiency in terms of total transmissions per search/broadcast and quantitatively analyze the information vs. efficiency trade-off in networks. The trade-offs are provided in Table I. We show the following results on broadcast efficiency for networks with varying levels of information.

\footnotetext{
${ }^{2}$ The parameter $n$ roughly corresponds to the density of the network.
} 


\begin{tabular}{|c|c|c|}
\hline Information type & Transmissions $T(n)$ & Congestion \\
\hline Zero Information & $c^{\frac{1}{M(n)}}, c>1$ & Heavy congestion about the source \\
\hline Source Quadrant Information & $\left(\frac{1}{M(n)}\right)^{5 / 2}$ & Moderate congestion about the source \\
\hline Transmission State Information & $\left(\frac{1}{M(n)}\right)^{2} \log \frac{1}{M(n)}$ & Low congestion throughout the network \\
\hline
\end{tabular}

TABLE I

TRADE-OFFS IN BROADCASTING - NETWORKS WITH LIMITED INFORMATION

(i) In networks with zero information, we present broadcast strategies based on random packet relaying. We show that exponentially large number of transmissions (of the order of $c^{\frac{1}{M(n)}}$, for some $c>1$ ) are required to ensure a transmission within a radius $M(n)$ of any given node at a unit distance from the source of the broadcast, to achieve a broadcast delay of $\Theta\left(\frac{1}{M(n)}\right)$. Moreover, we show that there are a large number of simultaneous transmissions in the region surrounding the source node, thus causing congestion in that area.

(ii) We consider networks with source quadrant information, and present a (sub)-broadcasting strategy based on packet forwarding that provides radial drift to the transmissions. We show that the outward spread of the transmissions reduce the broadcast redundancy. We show that only $\left(\frac{1}{M(n)}\right)^{5 / 2}$ transmissions are required to achieve a delay $D(n)=\Theta\left(\frac{1}{M(n)}\right)$ (however, with a possible advertisement radius that scales as $\left.A(n)=(M(n))^{\gamma}, \gamma<0.5\right)$. Equivalently, this scheme can be interpreted as a subbroadcasting strategy where at least one node in any region of radius $A(n)$ receives a broadcast message.

(iii) In networks with state information, we show that broadcast strategies can learn to inherently provide a radial drift to the transmissions. The broadcast strategies can use the state information to suppress transmissions by redundant nodes and advance the packets away from the source of the broadcast. By considering a strategy of suppression based on [9], we show that this implied radial drift suffices to achieve optimal broadcast delays with $T(n)=\left(\frac{1}{M(n)}\right)^{2} \log 1 / M(n)$, and negligible congestion throughout the network.

(iv) For the problem of spatial sampling in networks with local direction information, we present a randomized-tree based broadcast strategy that provides a lower transmission overhead. We show that we can sample on a "grid" of a given size $s(n) \sim \Omega\left(\frac{1}{\log \frac{1}{M(n)}}\right)$ (see Figure 3), as long as the "bin" size (the local advertisement radius) scales as $(M(n))^{\gamma}, \gamma<.49$. Such a sampling requires the number of transmissions to scale as $T(n)=\left(\frac{1}{M(n)}\right)^{\alpha}$, for a finite $\alpha$ that depends on $s(n)$.

Finally, we provide continuum model based simulations which support the analytical results obtained in the paper. From the above results we infer that broadcasting with very little geographic or state information is significantly more efficient than networks without any such "local" knowledge. While strategies with local state information can provide low transmission overheads and low congestion, the memory requirement scales linearly with the number of simultaneous broadcast messages. Further, such strategies also require nodes to compare the messages in their memory with every received broadcast. On the other hand, we note that strategies with geographic location information also provide substantial reductions in the number of transmissions, and the information requirements do not increase with simultaneous broadcasts. However, obtaining geographic information at the sensor nodes might require significant computation and/or hardware, such as GPS. In practice, these considerations can be used to tradeoff between memory, hardware and energy efficiency (number of transmissions).

\section{B. Related Work}

There has been considerable work on broadcasting and querying in sensor networks [22], [18], [2], [19], [13], [15], [4], [1], [12], [11], [16]. It was demonstrated in [13] that flooding based broadcasting/querying schemes such as [11], [12] cause many redundant transmissions leading to the broadcast storm problem. As discussed in [23], many of the broadcast schemes introduced to mitigate the "Broadcast storm" problem can be classified into the following categories:

(i) Probabilistic schemes such as [9], [14] in which nodes that receive the message rebroadcast with a fixed probability. In these schemes the nodes are assumed to have state information to remember previously received messages and utilize them to suppress secondary transmissions.

(ii) Location based schemes proposed in [13] where node transmission decisions are based on the expected area covered.

(iii) Neighbor knowledge based methods such as [2], [15], [19] where the location of the neighbors or the twohop neighbors are known. In [22], perfect information about the position of all nodes in the network is utilized to construct minimum energy broadcast trees, whereas in [2], the authors provide a construction for a similar tree based on local topology information. In [19], the two-hop neighbor information is utilized for building connected dominating sets that efficiently broadcast information.

Also, querying in sensor networks has been studied in papers such as [16], [5], authors in [1] propose random walks initiated by the source node and the destination node. They have shown that querying delay, transmission overheads can be reduced by spreading routing information through the network. This phenomenon has been quantitatively studied in [17].

Although many of the broadcast strategies previously discussed utilize some kind of local knowledge or state information, we note that a systematic analysis of the role of information in broadcasting, and the related trade-offs in the number of transmissions, delay and congestion, has not been explored previously. In this paper, we study a sequence of networks with varying levels of geographic and state information, 
and compare broadcast trade-offs through analytical methods. Further, we provide simulation results to validate the analytical studies.

\section{System DESCRIPTION}

\section{A. Network Model}

We consider a sensor network in which the sensor nodes are deployed over a two-dimensional planar region. Each of these sensor nodes are assumed to have a common circular transmission region and are connected to all other sensor nodes that lie within its transmission radius. The transmission radius is set to scale as $M(n)$, where $n$ is the scaling parameter. ${ }^{3}$ In this paper, we study broadcast strategies in dense networks in the large- $n$ regime, (where $n \rightarrow \infty$ ). The results of [8] show that for

$$
M(n)=\Omega\left(\sqrt{\frac{\log n}{n}}\right),
$$

the network formed by the collection of sensor nodes in a given region of finite area is asymptotically connected, and more importantly the number of nodes in the transmission radius of each node in that given region tends to infinity asymptotically. In this paper, we consider any $M(n)$ that scales as $O\left(\frac{1}{n^{p}}\right), p \in$ $\left(0, \frac{1}{2}\right)$, to model the growth of the network size relative to the radio range.

Motivated by the above results, in this paper we assume a continuum model of the sensor network, where any point in the radio range of a transmitting node $S$ can receive the packet transmitted by the node, and can act as a retransmission node. The neighbor set (nodes within the radio range) of any node $S$ in the sensor network is defined as

$$
\mathcal{N}_{S, M(n)}=\left\{X \in \mathbb{R}^{2}: d(X, S)<M(n)\right\},
$$

where the distance metric $d$ is the Euclidean distance. Thus, there is a one-to-one correspondence between nodes and their locations and the discretization effects due to node locations are ignored in the continuum model. However, as mentioned above, in densely connected sensor networks, the number of nodes within the radio range of any particular node increases to infinity [8]. Thus, the continuum model appears reasonable in this regime. We refer to [20] for a comparison of analytical results using a continuum model and simulation results with a discrete model with a dense network of nodes, which indicate that the discretization effects are not significant.

\section{B. Broadcast Model}

Querying and Information spreading, are both studied as a series of packet forwards in a sensor network. Since the transmissions in a wireless sensor network are inherently broadcast transmissions, we assume that whenever a node transmits a query, all nodes in its neighbor set can potentially receive it without error.

To broadcast a query "m", the originating node $S_{0}$ sends out a packet, to all its neighbors (in a single transmission) and requests a subset $\mathcal{S}_{1}$ of its neighbors to retransmit it. The repeated application of this process disseminates the information/query into the network. Let $S_{0}=\mathbf{0}$ be the position of the source node, i.e., the position of the node initiating the

\footnotetext{
${ }^{3}$ The quantity $n$ roughly corresponds to the density of nodes in the network.
}

query. The set $\mathcal{S}_{i}$ is the set of all points (nodes associated with the points) in the network that transmit the packet at the $i^{\text {th }}$ iteration of the process, or are the $i^{\text {th }}$ generation transmitters.

In this setup, we define the normalized broadcast delay, $D(n)$ as follows. Let $X$ be any given point (or the node at position $X)$, a unit distance away from the origin. We define

$$
D(n)=\inf \left\{i: d\left(\mathcal{S}_{i}, X\right)<M(n)\right\}
$$

where $d$ is the Euclidean distance metric. That is, the normalized delay $D(n)$ is defined as the iteration by which there is a transmission within the radio range of the given point/node $X$. Note that the 'unit' distance between the node and the origin is arbitrary. For any other distance $r$, the hop-delay can be scaled accordingly. Thus, we define $D(n)$ as the hop count of the minimum hop path from the source to reach any arbitrarily chosen node $X$ that is a unit distance away.

We note that in this definition, the medium access delay has been ignored, and delay is measured only in terms of the hop count. We note that the actual packet delay can be decomposed into the hop count delay and the MAC delay. By suitably scaling the packet size (see [6] for this approach), we can achieve a MAC delay that is order-wise smaller than the hop-count delay. Thus, in this regime, the hop-count will be representative of the packet delay. Even if such a packet-scaling was not employed, the delay with two broadcasting schemes can be compared using a pair of metrics: (i) hop-count delay, and (ii) the "local" congestion about a transmitting node (i.e., the number of transmissions that occur in a spatial region) which clearly plays an important part in determining MAC delay. Thus, in addition to small hop-count, a good broadcasting scheme will mitigate local congestion. This observation motivates us to later consider "branching" based schemes where the number of transmissions progressively increases with radial distance from the source, and has an (order-wise) same hop-count an more "concentrated" broadcasting schemes.

In the case of querying, the normalized delay corresponds to the number of iterations required to reach any given point that is located a unit distance away from the source node. In the context of information spreading, the normalized delay corresponds to the iterations required to spread the information to a randomly chosen point which is a unit distance away from the source. Thus, the above definition of delay allows us to study the symmetric problems of information spreading and querying within the same framework.

We also define the transmission overhead $T(n)$ as the total number of transmissions by the iteration $D(n)$, i.e.

$$
T(n)=\sum_{k=1}^{D(n)}\left|\mathcal{S}_{k}\right|,
$$

where $|$.$| denotes the cardinality of the set. Conventional flood-$ ing based strategies achieve a broadcast delay of $\Theta\left(\frac{1}{M(n)}\right)$ hops in densely connected networks, as the minimum distance of order $M(n)$ is covered in each iteration along all directions. In order to compare the various broadcast strategies, we constrain the broadcast strategies in all network models to achieve order-wise optimal hop-delays. Further, if the delay is $\frac{K}{M(n)}, K<\infty$, for any arbitrarily picked node, the broadcast can be efficiently terminated by setting TTL values in the broadcast packets appropriately. Thus, in the rest of the paper, we only consider strategies that have a delay of $\Theta\left(\frac{1}{M(n)}\right)$. 


\section{BROADCASTING IN NETWORKS WITH ZERO INFORMATION.}

In this section, we study the energy-delay trade-offs of broadcasting, in networks with zero information. We assume that the nodes in the network do not have any geographic or state information. That is, the nodes have no knowledge of the locations of their neighbors or of the broadcast source, and are incapable of remembering previous messages or transmission routes. Since nodes have no state information, decisions to retransmit a received message are made at the time of arrival of the message. Thus, it is possible for the same message to be received and transmitted multiple times by a node.

To broadcast information in such networks very limited capability, we employ a simple broadcast strategy based on random packet forwarding, that requires no state or geographic information. In this scheme, each transmitting node selects only one retransmitting node randomly from its neighbor set (the nodes within the radio range $M(n)$ ), and requests the node to retransmit it. We study these "random walk" based schemes, as they are a sequence of simple communication operations and representative of broadcast strategies possible in networks with no information.

As discussed earlier in Section II, to compare the different broadcast schemes, we require the normalized broadcast delay, $D(n)=\Theta\left(\frac{1}{M(n)}\right)$. By randomly forwarding a single message, it may not be possible to achieve the required normalized delay and hence we initiate multiple broadcasts of the same message, corresponding to independent parallel random walks. That is, we originate $R(n)$ independent copies of the same broadcast message at the source node, and propagate each message by random packet forwarding.

To analyze the energy efficiency of the broadcast strategy, we choose a random node that is a unit distance away from the source node, and compute the total number of transmissions $T(n)$ that are required to ensure that the message is received by the chosen node, within $\Theta\left(\frac{1}{M(n)}\right)$ iterations. The energy efficiencies are studied in terms of the number of broadcasts.

\section{A. Random Packet Forwarding}

The packet forwarding based broadcast, with multiple copies of the broadcast message, has a simple communication structure. The source node transmits $R(n)$ independent copies of the broadcast message, i.e., for every copy of the message, the source node picks another sensor node randomly from its neighbor set for retransmission. Every transmitting node has only one offspring node, and only one transmission per query/message occurs at every iteration. That is, at the $i^{\text {th }}$ iteration, the position of the transmitting node for the $k^{t h}$ copy of the message is

$$
S_{i}^{k}=S_{i-1}^{k}+X_{i}^{k}
$$

where denotes $S_{l}^{k}$ the position of the $k^{\text {th }}$ random walk after $l$ iterations and $X_{i}^{k}$ is the random displacement from the node transmitting copy $k$ at iteration $i-1$. We assume that $X_{i}^{k}$ are i.i.d random variables, with a common distribution $\mu$. Since no geographic location information is available, we assume that the next hop nodes are chosen uniformly randomly from the neighbor set of each transmitter and assume that the distribution $\mu$ is uniformly distributed over the compact set $B_{M(n)}(0)$, where $B_{r}(x)$ denotes a ball of radius $r$ around $x$.
Assumption 3.1: We assume that $\mu$ has variance $\sigma_{\mu}^{2}(n)>0$, a compact support $B_{M(n)}(0) \subset \mathbb{R}^{2}$.

We use the following notation for $\mathrm{n}$-fold convolutions of $\mu$,(i.e., the distribution of $n$ random variables with distribution $\mu)$

$$
\mu_{(n+1)}(A):=\int \mu_{(n)}(A-x) \mu(d x), \quad n \in \mathbb{N},
$$

where $\mu_{(1)}:=\mu$.

Under the above model for a network with Zero Information, we show that the number of transmissions increasing exponentially with $\frac{1}{M(n)}$ (the network diameter in hops), are necessary and sufficient to ensure an optimum broadcast delay. The following theorem shows that exponentially large number of transmissions are necessary to achieve a delay $D(n)=$ $\Theta\left(\frac{1}{M(n)}\right)$, using the broadcasting strategy discussed earlier in this section. We show that, even if the number of paths are exponentially large, the probability that none of the paths reach the radio range of the node within $\Theta\left(\frac{1}{M(n)}\right)$ steps is high.

Theorem 3.1: For any given $K<\infty$, there is a $c>1$ such that for $R(n)=c^{\frac{1}{M(n)}}$,

$$
\mathbb{P}\left(\bigcap_{l=1, \ldots, R(n)} S_{k=1, \ldots, \frac{K}{M(n)}}^{l} \notin B_{\epsilon}(x)\right) \longrightarrow 1 .
$$

for some $\epsilon>0$.

Proof:

$$
\begin{array}{r}
\mathbb{P}\left(\bigcap_{l=1, \ldots, R(n)} S_{k=1, \ldots, \frac{K}{M(n)}}^{l} \notin B_{\epsilon}(x)\right) \\
=\mathbb{P}\left(\bigcap_{k=1, \ldots, \frac{K}{M(n)}} S_{1}^{k} \notin B_{\epsilon}(x)\right)^{R(n)}, \\
\geq\left(1-\sum_{k=1}^{\frac{K}{M(n)}} \mathbb{P}\left(S_{k}^{l} \in\left(B_{\epsilon}(x)\right)\right)\right)^{R(n)}, \\
=\left(1-\sum_{k=\frac{1}{M(n)}} \mathbb{P}\left(S_{k}^{l} \in\left(B_{\epsilon}(x)\right)\right)\right)^{R(n)}, \\
=\left(1-\frac{K}{M(n)} \mathbb{P}\left(S_{\frac{K}{M(n)}}^{l} \in\left(B_{\epsilon}(x)\right)\right)\right)^{R(n)} .
\end{array}
$$

Equation (9) follows since the maximum distance covered in a hop is only $M(n)$.

Note that

$$
\mathbb{P}\left(S_{\frac{K}{M(n)}}^{1} \in B_{\epsilon}(x)\right) \leq \mathbb{P}\left(S_{\frac{K}{M(n)}}^{1} \in B_{1-\epsilon}^{c}(0)\right)
$$

By Chernoff's bound, $\mathbb{P}\left(S_{n}^{1} \in B_{1-\epsilon}^{c}(0)\right) \leq e^{-n I(\delta)}$, for small $\delta>0$, where $I$ is the rate function associated with the random variables. Hence,

$$
\begin{array}{r}
\mathbb{P}\left(\bigcap_{l=1, \ldots, R(n), \quad{ }_{k=1, \ldots, \frac{K}{M(n)}}} S_{k}^{l} \notin B_{\epsilon}(x)\right) \\
\geq\left(1-\frac{K}{M(n)}\left(e^{-\frac{1}{M(n)} I(\delta)}\right)\right)^{R(n)} .
\end{array}
$$




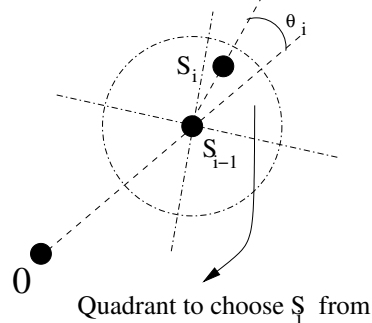

(i) Choice of Quadrant

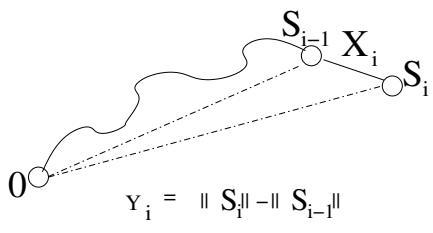

(ii) Radial Progress
Fig. 4. Random packet forwarding with knowledge of source location, and local quadrants

Let $R(n)=c^{\frac{1}{M(n)}}$ for any $c<e^{-I(\delta) / 2}$ then, the R.H.S term tends to 1 as $n \rightarrow \infty$.

Thus, the total number of required transmissions, $T(n)=$ $c^{\frac{1}{M(n)}} \times \frac{2}{M(n)}$, grows exponentially with the network diameter $\frac{1}{M(n)}$.

We now show in following theorem that it is also sufficient to have exponentially large number of transmissions, to achieve a delay of $\Theta\left(\frac{1}{M(n)}\right)$, using the broadcasting strategy discussed earlier in this section.

Theorem 3.2: Consider $R(n)$ random walks starting from $S_{0}=\mathbf{0}$ and any given point $x=\left(x_{1}, x_{2}\right)$ on the boundary of the compact ball $B_{1}(0)$. Then, there exists a $c<\infty$ such that for $R(n) \geq c^{\frac{1}{M(n)}}$,

$$
\min _{k \in 1, \ldots, R(n)}\left\|S_{\frac{2}{M(n)}}^{k}-x\right\| \leq M(n) . \quad \text { (prob.) }
$$

That is, there exists a random walk that is arbitrarily close to $x$, after $\frac{2}{M(n)}$ iterations.

Proof: The proof is omitted here for brevity, and is available in [21].

Remark 3.1: Thus, the results in this section indicate that exponentially large number of transmissions are necessary and sufficient to successfully broadcast in sensor networks with no geographic or state information. We also note that, by employing multiple queries/messages, the number of transmissions by nodes close to the source node increases linearly with the $R(n)$. In networks with Zero Information, this translates to an exponentially large number of transmissions in a small area (areas of the size of the radio range) close to the source node, causing network congestion.

\section{BRoAdcasting in Networks With SOURCE QUADRANT INFORMATION}

In this section, we study the efficiency of broadcasting in networks with source quadrant information and compute the number of transmissions required to obtain a normalized delay of $\Theta\left(\frac{1}{M(n)}\right)$. We assume that the nodes have only a local notion of four directions which are not necessarily common to all nodes. That is, the nodes are capable of grouping their neighbors into four different quadrants, where the orientations of the quadrants are chosen independently by different nodes. To model this, we assume that the orientations of the quadrants are uniformly distributed between angles 0 and $2 \pi$, and are chosen independently of the local quadrants at other nodes. We also assume that there is some data embedded in a packet's header that enables an intermediate node to infer coarse geographic source location w.r.t its local quadrants. This could be implemented, for instance, if the packet has the source location embdded in its header and nodes have possibly faulty GPS (see [20]). Thus, the nodes are assumed to have Source Quadrant Information. However, we assume that the nodes have no state information i.e., they are incapable of remembering any previous transmissions or messages.

To broadcast in networks with limited geographic information, we study broadcasting strategies similar to the schemes presented in Section III. The broadcast strategy follows the random packet forwarding model, but utilizes the location information to direct the packets radially away from the source node, reducing the broadcast redundancy. We again use the multiple independent query model to achieve a normalized delay of $\Theta\left(\frac{1}{M(n)}\right)$. The broadcast strategy is as follows:

1) The source node picks $R(n)$ neighbors uniformly randomly (i.e., $R(n)$ points independently chosen from its neighbor set), and sends the broadcast message to them.

2) Each of the nodes, on receiving a request to transmit, retransmit the message and choose exactly one neighbor from the "local" quadrant opposite to the source's quadrant, and request that neighbor to retransmit the message.

We show that this scheme provides a sub-broadcasting strategy where at least one node in any region of radius $A(n)=$ $(M(n))^{\gamma}, \gamma<0.5$ receives a broadcast message.

\section{A. Broadcast Model with Source Information}

Let the source node be at $\mathbf{0}$, and consider any given copy (indexed by $k$ ) of the broadcast message. We denote the transmitting node at the $i-1^{t h}$ iteration to be $S_{i-1}^{k}$. Since the Source Quadrant Information is available to all nodes, the transmitting node for the $i^{t h}$ iteration $(i \geq 2), S_{i}^{k}$, is chosen uniformly from the opposing quadrant. Let us denote the offset angle (from the line joining the source and the node) by $\theta_{i}^{k}$, as shown in Figure 4(i).

The radial progress in the $i^{\text {th }}$ jump is defined as the random variable $Y_{i}^{k}$, where $Y_{i}^{k}=\left\|S_{i}^{k}\right\|-\left\|S_{i-1}^{k}\right\|$. Moreover, as the initial direction of transmission is uniformly distributed over $[0,2 \pi], S_{i}^{k}$ are also angularly uniformly distributed (see [21] for additional details). In [20], it has been shown that $Y_{i}^{k}$ are 'well-approximated'4 by $\left\|X_{i}^{k}\right\| \cos \theta_{i}^{k}$, a sequence of i.i.d random variables, having compact support in $[0, M(n)] \subset$ $\mathbb{R}$. Motivated by the above approximation, in this section, we model the radial progress at each step by i.i.d. random variables.

Consider $R(n)$ independent messages originating at $S_{0}^{k}=$ 0. The position of the $k^{t h}$ walk is given by $\left(Z_{i}^{k}, \phi_{i}^{k}\right.$ ) (in polar coordinates), where $Z_{i}^{k}$ be the radial distance traveled in $i$ iterations of the $k^{t h}$ random walk. The angle $\phi_{i}^{k}$ is uniformly distributed in $[0,2 \pi]$ and independent of $Z_{i}^{k}$ and also of $\phi_{m}^{n}$ if $n \neq k$. This follows, because the initial angle $\theta_{1}^{k}$ is chosen uniformly over $[0,2 \pi]$, independent of any other quantity (see [21]). The radial displacement is given by

$$
Z_{i}^{k}=Z_{i-1}^{k}+Y_{i}^{k}, \quad i \geq 2
$$

where $Y_{i}^{k}$ are i.i.d random variables, with support $[0, M(n)] \subset$ $\mathbb{R}$ and $E\left(Y_{1}\right)=d M(n), d>0$. Also, $Z_{1}^{k}=Y_{1}^{k}$,

\footnotetext{
${ }^{4}$ The approximation is in the sense that the error in the source-to-destination path length is vanishingly small, see Lemma 3.2 and Theorem 3.1 in [20] for additional details.
} 
where $Y_{1}^{k}=X_{1}^{k} \cos \tilde{\theta}_{1}^{k}$ and $\tilde{\theta}_{1}^{k}$ is chosen as a independent, identically distrbuted random variable as $\theta_{2}^{k}$. Note that the actual radial displacement in the first hop is actually $X_{1}^{k}$; however, the above approximation in the first hop leads to a vanishingly small source-to-destination path length error, which can handled by a receiver advertisement radius, see Remark 4.1. We define the distribution of $Y_{i}^{k}$ on $[0, M(n)]$, by $\nu$.

Under these conditions, the following theorem provides an upper bound on the number of transmissions required to ensure a delay of $\Theta\left(\frac{1}{M(n)}\right)$. We require the following corollary of the result in [7] Thm.1, pg. 533, on the concentration of the distribution about its mean.

Lemma 4.1: We assume that $\psi$ is a probability measure with mean $t$, variance $\sigma_{\psi}^{2}>0$, and a compact support $B \subset \mathbb{R}$. Then, for some $K<\infty$, there exists an $N_{0}$ such that

$$
\psi_{(n)}\left(\left[n t-\frac{\varepsilon}{2}, n t+\frac{\varepsilon}{2}\right]\right) \geq \frac{K}{\sqrt{n}}, \quad \forall n>N_{0} \in \mathbb{N} .
$$

Theorem 4.1: Consider $R(n)$ random walks starting from $S_{0}=\mathbf{0}$ and any given point $x=\left(1, \theta^{*}\right)$ on the boundary of the compact ball $B_{1}(0)$. Let $c_{1}=\frac{1}{d}$ Then, for $R(n) \geq \frac{1}{M(n)}^{\alpha}$, $\alpha>3 / 2$,

$$
\min _{k \in 1, \ldots, R(n)}\left\|S_{\frac{c_{1}}{M(n)}}^{k}-x\right\| \leq M(n) . \quad \text { (prob.) }
$$

Proof: Consider the probability

$$
\mathbb{P}\left(\bigcap_{k \in\{1, \cdots, R(n)\}} S_{\frac{c_{1}}{M(n)}}^{k} \notin B_{M(n)}(x)\right) .
$$

Note that, as the paths are independent and identically distributed,

$\mathbb{P}\left(\bigcap_{1 \leq k \leq R(n)} S_{\frac{c_{1}}{M(n)}}^{k} \notin B_{M(n)}(x)\right)=\mathbb{P}\left(S_{\frac{c_{1}}{M(n)}}^{1} \notin B_{M(n)}(x)\right)^{R(n)}$

By definition, $S_{\frac{c_{1}}{M(n)}}^{1}=\left(Z_{\frac{c_{1}}{M(n)}}^{1}, \theta_{\frac{c_{1}}{M(n)}}^{1}\right)$, and hence the probability

$$
\begin{aligned}
& \mathbb{P}\left(S_{\frac{c_{1}}{M(n)}}^{1} \notin B_{M(n)}(x)\right) \leq \\
& \mathbb{P}\left(\left\{Z_{\frac{c_{1}}{M(n)}}^{1} \notin[1-M(n) / 4,1+M(n) / 4]\right\}\right. \\
&\left.\cup\left\{\theta_{\frac{c_{1}}{M(n)}}^{1} \notin\left[\theta^{*}-\frac{M(n)}{4}, \theta^{*}+\frac{M(n)}{4}\right]\right\}\right) \\
&=1- {\left[\mathbb{P}\left(\left\{Z_{\frac{c_{1}}{M(n)}}^{1} \in[1-M(n) / 4,1+M(n) / 4]\right\}\right)\right.} \\
&\left.\times \mathbb{P}\left(\left\{\theta_{\frac{c_{1}}{M(n)}}^{1} \in\left[\theta^{*}-\frac{M(n)}{4}, \theta^{*}+\frac{M(n)}{4}\right]\right\}\right)\right],
\end{aligned}
$$

by the independence of $Z_{\frac{c_{1}}{M(n)}}^{1}$ and $\theta_{\frac{c_{1}}{M(n)}}$.

By applying Lemma 4.1 to the random variables $Z_{i}^{1}$, there exists a $k_{1}>0$, such that

$\mathbb{P}\left(\left\{Z_{\frac{c_{1}}{M(n)}}^{1} \in[1-M(n) / 4,1+M(n) / 4]\right\}\right) \geq k_{1} \sqrt{M(n)}$,

as $E\left(Z_{\frac{c_{1}}{M(n)}}^{1}\right)=1$. By the uniform distribution of $\theta_{\frac{c_{1}}{M(n)}}^{1}$, it follows that

$$
\mathbb{P}\left(\left\{\theta_{c_{1}} \in\left[\theta^{*}-\frac{M(n)}{4}, \theta^{*}+\frac{M(n)}{4}\right]\right\}\right)=\frac{M(n)}{4 \pi} .
$$

By Equations 15,16, and for some $k_{2}>0$

$$
\mathbb{P}\left(S_{\frac{c_{1}}{M(n)}}^{1} \notin B_{M(n)}(x)\right) \leq 1-k_{2} M(n)^{\frac{3}{2}} .
$$

For $R(n)=\frac{1}{M(n)}^{\alpha}, \forall \alpha>\frac{3}{2}$,

$$
\begin{aligned}
& \mathbb{P}\left(\bigcap_{k \in\{1, \cdots, R(n)\}} S_{\frac{c_{1}}{M(n)}}^{k_{(n)}} \notin B_{M(n)}(x)\right) \\
& \quad \leq\left\{1-k_{2} M(n)^{\frac{3}{2}}\right\}^{R(n)} \rightarrow 0 .
\end{aligned}
$$

Theorem 4.1 thus follows.

Remark 4.1: Thus, the total number of transmissions $T(n)=\left(R(n) * \frac{c_{1}}{M(n)}\right)$ is less than $\left(\frac{1}{M(n)}\right)^{\alpha}$ for any $\alpha>\frac{5}{2}$. The results demonstrate that it is sufficient for $\left(\frac{1}{M(n)}\right)^{5 / 2}$ transmissions to broadcast to any randomly chosen point that is a unit distance away from the source, with local geographic knowledge, even without any suppression of transmissions. However, we note that the broadcast strategy causes a polynomially large (of order $\frac{1}{M(n)}^{3 / 2}$ ) number of transmissions around the source node, causing significant congestion, although the congestion is substantially lower, compared to broadcasting with Zero Information, where the number is exponentially large. Also, we note that this approach could possibly require nodes to locally advertise with a radius that scales as $A(n)=(M(n))^{\gamma}, \gamma<0.5$ (see Section VI for additional discussion). This is to compensate for the error in the approximation where we have assumed that the radial displacements are i.i.d. (see [20], [21] for additional details). Equivalently, this scheme provides a sub-broadcasting strategy where at least one node in any region of radius $A(n)$ receives a broadcast message.

\section{BROAdCASTING With Limited STATE INFORMATION}

In this section, we analyze broadcasting in networks with limited state information. We assume that the nodes in the network are capable of remembering previously received messages and their decision to transmit or to not transmit the received message. However, we assume that the nodes have no knowledge of the position of the neighbors or the source node. In such networks with very little state information, and no location information, we study broadcast strategies that possess broadcast delays of $D(n)=\Theta\left(\frac{1}{M(n)}\right)$ and compute the number of transmissions required to achieve the order-wise optimal delays. The broadcast scheme we study is a variation of the gossip algorithm presented in [9] where a node decides to retransmit the broadcast message with a probability $p$, upon the first arrival of the message. The broadcast algorithm we employ is described below.

1) In the first iteration, the source node $S_{0}$ transmits the message ' $\mathrm{m}$ ', to all its neighbors, and chooses $C \log \frac{1}{M(n)}$ nodes randomly from its neighbor set, and requests them to retransmit the message.

2) In the next iteration, the chosen nodes transmit their message and choose $C \log \frac{1}{M(n)}$ nodes randomly from their neighbor sets, but nodes that have received the previous broadcast of the message ignore all subsequent broadcasts of the same message. Thus, nodes chosen from regions that had previously heard the message do not transmit. 


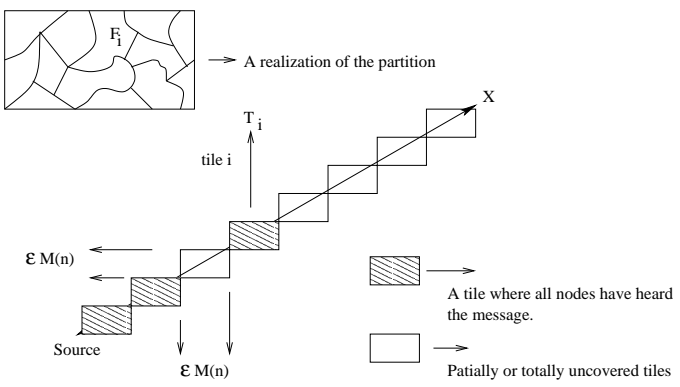

Fig. 5. Branching in Sensor nodes.

3) The process is repeatedly iterated to spread the query over the network.

Thus, the algorithm employs the state information to suppress redundant transmissions in regions that have previously received the broadcast message. For this "location-less" broadcast scheme, we show that the delay $D(n)=\Theta\left(\frac{1}{M(n)}\right)$, while the total number of transmissions are $O\left(\frac{1}{M(n)}^{\alpha}\right)$, where $\alpha>2$.

In the following theorem, we first prove that the broadcast algorithm discussed previously achieves a delay of $\Theta\left(\frac{1}{M(n)}\right)$. We show this, by choosing any node $X$, that is a unit distance away from the Source node $S_{0}$ and demonstrating that there is a transmission within the radio range of that given node within $\Theta\left(\frac{1}{M(n)}\right)$ iterations.

By our notations in Section II, we define $\mathcal{S}_{i}$ to be the set of transmitters in iteration $i$ and $\mathcal{P}_{i}$ to be the set of all transmitters till iteration $i$.

Theorem 5.1: Let $S_{0}=0, X$ be any given point such that $\left\|X-S_{0}\right\|=1$. Then, for some $\epsilon>0$, there exists a $0<C_{\epsilon}<$ $\infty$, such that for $C=C_{\epsilon}$,

Proof:

$$
\min _{Y \in \mathcal{P} \frac{1}{\epsilon M(n)}}\|X-Y\| \leq M(n) \quad \text { (prob.) }
$$

Consider tiles of size $\epsilon M(n) \times \epsilon M(n)$ about the line connecting the source node and $X$, as in Figure 5. We choose $\epsilon>0$ such that a transmission (of range $M(n)$ ) in any tile covers the adjacent tiles as well (it can be seen that for any $\epsilon<\frac{1}{3}$, this condition is satisfied). A tile is defined to be 'covered' if all nodes within the tile have received the broadcast message; else it is defined to be 'uncovered'. Let $A_{t}$ be the event $\left\{\right.$ Tile $T_{t}$ covered by time $\left.t\right\}$ and let the event $E_{t}$ be the event $\left\{\right.$ Some node in Tile $T_{t}$ was picked as a transmitter $\}$. We require the following lemma.

Lemma 5.1: The probability

$\mathbb{P}\left(E_{t}^{c} / A_{t}\right)=\mathbb{P}\left(\right.$ No transmissions in tile $\left.T_{k} \mid A_{t}\right) \leq M(n)^{\frac{C \epsilon^{2}}{\pi}}$

Proof: Let $\mathcal{W}$ be any partitioning of the tile $T_{t}$. Let the partition $\mathcal{W}$ be the union of disjoint sets $F_{i}, i=1, \cdots, f(n)$, where the disjoint sets $F_{i}$ correspond to the incrementally covered regions of the tile $T_{t}$, over different transmissions (see Figure 5 for an illustration). Let $l\left(F_{i}\right)$ denote the fraction of the area of $F_{i}$ in the tile, with $\sum_{i=1}^{f(n)} l\left(F_{i}\right)=1$. Then,

$$
\mathbb{P}\left(E_{t}^{c} \mid A_{t}\right)=\int \mathbb{P}\left(E_{t}^{c} \mid A_{t}, \mathcal{W}\right) d \mu_{A_{t}}(\mathcal{W}),
$$

where $\mu_{A_{t}}(\mathcal{W})$ is the probability that the partition $\mathcal{W}$ was created by the transmission process. We now derive an uniform upper bound on $\mathbb{P}\left(E_{t}^{c} \mid A_{t}, \mathcal{W}\right)$ (which does not depend on $\mathcal{W}$ ), and hence, provide an upper bound on L.H.S of (21).

Since we choose $C \log M(n)$ nodes uniformly from an area of $\pi(M(n))^{2}$, the probability

$$
\begin{aligned}
\mathbb{P}\left(E_{t}^{c} \mid A_{t}, \mathcal{W}\right) & =\prod_{i=1}^{f(n)}\left(1-\frac{l\left(F_{i}\right) \epsilon^{2}}{\pi}\right)^{C \log \frac{1}{M(n)}}, \\
& =\prod_{i=1}^{f(n)} M(n)^{\left(C \log \left(\frac{1}{\left.1-\frac{l\left(F_{i}\right) \epsilon^{2}}{\pi}\right)}\right)\right.} \\
& \left.=M(n)^{-C\left(\sum_{i=1}^{f(n)} \log \left(1-\frac{l\left(F_{i}\right) \epsilon^{2}}{\pi}\right)\right.}\right)
\end{aligned}
$$

As $M(n)<1$, we now have from (22)

$$
\mathbb{P}\left(E_{t}^{c} \mid A_{t}, \mathcal{W}\right) \leq M(n)^{\left[C \beta^{*}\right]}
$$

where

$$
\begin{aligned}
\beta^{*}=- & \max _{x_{i}: 1 \leq i \leq f(n)} \sum_{i=1}^{f(n)} g\left(x_{i}\right), \\
& \text { s.t. } \sum_{i=1}^{f(n)} x_{i}=1, \quad x_{i} \in[0,1], \\
g(x)= & \log \left(1-\left(\epsilon^{2} / \pi\right) x\right), \quad x \in[0,1] .
\end{aligned}
$$

It can be directly computed to show that $g(x)$ is a negative concave function with $g(0)=0, g(1)=\log \left(1-\left(\epsilon^{2} / \pi\right)\right)$. By using Lagrange Multipliers, it can be shown that for each fixed $f(n)$, the maximum is achieved when $x_{i}=\frac{1}{f(n)}$, for all $i$. Thus,

$$
\beta^{*}=-\max _{f(n)} f(n) g\left(\frac{1}{f(n)}\right) .
$$

Further, we have $\log \left(1-\left(\epsilon^{2} / \pi\right)\right) \leq-\left(\epsilon^{2} / \pi\right)$, and hence, $\beta^{*} \leq\left(\epsilon^{2} / \pi\right)$. The result now immediately follows.

Now, the probability that the tile $T_{t+1}$ was covered by time $t+1$

$$
\begin{aligned}
\mathbb{P}\left(A_{t+1}\right) & \geq \mathbb{P}\left(A_{t} \cap E_{t}\right), \\
& =\mathbb{P}\left(A_{t}\right) \mathbb{P}\left(E_{t} / A_{t}\right), \\
& =\mathbb{P}\left(A_{t}\right)\left[1-\mathbb{P}\left(E_{t}^{c} / A_{t}\right)\right] .
\end{aligned}
$$

Note that the inequality in (25) is due to the fact that the event $A_{t} \cap E_{t}$ implies $A_{t+1}$, by construction. Utilizing Lemma 5.1 in (27),

$$
\begin{aligned}
\mathbb{P}\left(A_{t+1}\right) & \geq \mathbb{P}\left(A_{t}\right)\left(1-(M(n))^{C \epsilon^{2} / \pi}\right) \\
& \geq\left(1-(M(n))^{C \epsilon^{2} / \pi}\right)^{t}
\end{aligned}
$$

Hence, it follows that

$$
\mathbb{P}\left(A_{\frac{1}{M(n)}}\right) \geq\left(1-(M(n))^{C \epsilon^{2} / \pi}\right)^{\frac{2}{M(n)}} \rightarrow 1,
$$

for $\frac{C \epsilon^{2}}{\pi}>1$. Thus it is seen that by iteration $\frac{K}{M(n)}$, the tile $T_{\frac{K}{M(n)}}$ is covered with high probability.

By our construction, we see that in any tile $\mathrm{T}$, the number of transmissions is no greater than $C \log 1 / M(n)$. Since the total 


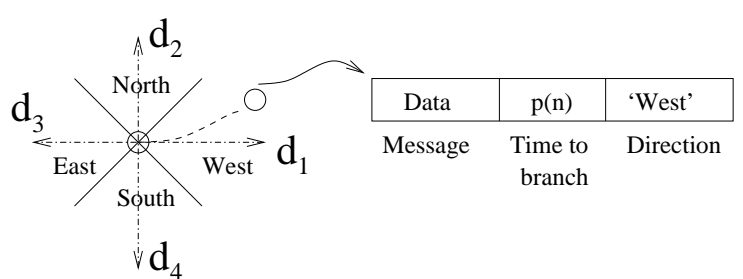

Fig. 6. Branching in Sensor networks with Local Direction Information

number of tiles is no greater than $K\left(\frac{1}{M(n)}\right)^{2}$, the total number of transmissions $T(n) \leq K_{1}\left(\frac{1}{M(n)}\right)^{2} \log 1 / M(n)$.

Remark 5.1: The results in this section demonstrate that "state information" in the networks can be utilized to simultaneously reduce the number of transmissions, and to distribute the transmissions more uniformly over the network. The proof in this sections show that the state information inherently provides a linear drift, emphasizing the role of suppression in efficient broadcasting. Further, the results can be extended to show that the branching algorithm can spread information uniformly in a two dimensional region. Moreover, uniformly spaced transmissions considerably reduce the congestion in the network.

\section{BROADCASTING OVER A LATTICE WITH LOCAL DIRECTION INFORMATION}

In this section, we study the problem of broadcasting to a set of spatially uniform nodes (lattice points) in networks where nodes have no "state" or geographic information, but only a rudimentary sense of local direction. That is, each sensor node in the network has an approximate sense of 'East', 'West', 'North' and 'South', formally defined in VI.A. Necessity for such a broadcasting scheme could arise when a spatially uniform sample of an underlying physical process is required by an application at the source node (see figure 3 ). For example, a sensor network deployed for measuring air quality might require measurements from the sensor network sampled uniformly over the deployed region; and thus, will need to send a query/message to the appropriate subset of nodes. We examine if such queries/messages can be broadcast efficiently with the availability of "local direction" information, and propose a random tree based broadcast protocol that utilizes the local information to spread messages over the network.

Under this broadcast scheme, we compute the number of transmissions required to reach a circular advertisement region of radius $A(n)$ about the destination node (a lattice point that is a unit distance away) within a delay of $\Theta\left(\frac{1}{M(n)}\right)$. We assume a lattice grid with a lattice spacing of $\frac{1}{\log \log 1 / M(n)}$. We show that under the strategy described below, that only $\left(\frac{1}{M(n)}\right)^{\gamma}, \forall \gamma>1$ transmissions are required to reach an circular advertisement region of radius $(M(n))^{\alpha}, \alpha<\frac{1}{2}$. We note that the choice of a lattice spacing of $\frac{1}{\log \log 1 / M(n)}$ in our proof is for notational ease. Our results can be immediately extended for any lattice spacing that is $\Omega\left(\frac{1}{\log 1 / M(n)}\right)$; however, the number of transmissions will then scale as $\left(\frac{1}{M(n)}\right) \bar{\gamma}$, for some finite $\bar{\gamma}>1$ (i.e., there will be a polynomial increase in the number of transmissions).

\section{A. Broadcast and Network Model}

We assume that sensor nodes in the network have an approximate knowledge about four antipodal directions $d_{(1)}, d_{(j)}, d_{(-1)}, d_{(-j)}$, In particular, the transmitting nodes have a local estimate of four antipodal directions $\bar{d}_{(1)}, \bar{d}_{(j)}, \bar{d}_{(-1)}, \bar{d}_{(-j)}$, such that for all $l \in\{1, j,-1,-j\}$

$$
E\left(d_{(l)} \cdot \bar{d}_{(l)}\right)=c, c>0 \text { and } E\left(\bar{d}_{(l)}\right)=d_{(l)} .
$$

In other words, we assume that the direction estimates are unbiased and with a positive projection. We note that the expected projection could be differ between directions, however we choose a uniform projection in all directions for notational simplicity.

We also assume that the packet contains information on the direction of travel, and a counter, to keep track of the number of hops traveled by a packet (Figure 6). Without loss of generality, we formally define the four directions to be $d_{(1)}=(1,0), d_{(j)}=(0,1), d_{(-1)}=(-1,0)$ and $d_{(-j)}=$ $(0,-1)$ (See Figure 6$)$. Thus, in a transmission by a node $\mathbf{x}$ along the direction $d_{i}$, the distance traveled in that transmission is a random variable $X$, with support $[0, M(n)] d_{i} \subset \mathbb{R}^{2}$, and $E(X)=c M(n) d_{i}, c>0$.

For networks with local direction information, the randomized tree (branching walk) based broadcast strategy is performed as follows (see Figure 7).

1) The source node $S_{0}=\mathbf{0}$ transmits a query to a randomly chosen retransmission node in each direction. The packets contain the data, the direction in which they were sent, and the Time to Branch(TTB) counter is set to $p(n)$ (See Figure 6).

2) The retransmission nodes check the packet's TTB counter. If $T T B=0$, then the retransmission node transmits one query each to the two orthogonal directions to the previous step, and sets $T T B=p(n)$, in the newly created query packets. If $T T B>0$, then $T T B$ value alone is changed to $T T B-1$, and the packet is retransmitted along the same direction.

Since the nodes create two queries at every branching, the spatial distribution of the query can be studied as a process indexed by a binary tree. Consider a query sent by the source node along the direction $d_{i}$. Let $\Gamma$ denote an infinite binary tree, where the vertices correspond to the queries generated by repeated branching of the initial query. Let $\Gamma_{(l, k)}$ denote the query at the $k$ th vertex at depth $l$, with $l \in \mathbb{N}$, and $k \in$ $J_{l}:=\left\{0,1, \ldots, 2^{l-1}-1\right\}$. Let $Z_{k}^{l}$ be the position of the query $\Gamma_{(l, k)}$, just before the $i+1^{t h}$ branching. Then,

$$
Z_{k}^{l}=Z_{\left[\frac{k}{2}\right]}^{l-1}+Y_{k}^{l}
$$

where $Y_{k}^{l}$ is the random distance traveled by the query after its $l^{\text {th }}$ branching. Hence, the random variable has a support $[0, p(n) M(n)] d_{i}$ and $E\left(Y_{k}^{l}\right)=c p(n) M(n) d_{i}$, where $d_{i}$ is the direction of travel of the query. As defined in Section II, we denote by $\mathcal{S}_{i}$, the set of transmitters in the $i$ th iteration.

Under the model discussed above, we show that the number of transmissions to reach a circular advertisement region of radius $A(n)=M(n)^{\alpha}, \alpha<\frac{1}{2}$ about any given point $x=\left(1, \theta^{*}\right)$ (in polar coordinates), with a normalized delay of $\Theta\left(\frac{1}{M(n)}\right)$ is $\frac{1}{M(n)}^{\gamma}, \forall \gamma>1$. That is, we show that the number of transmissions $T(n)$ is only marginally greater than 


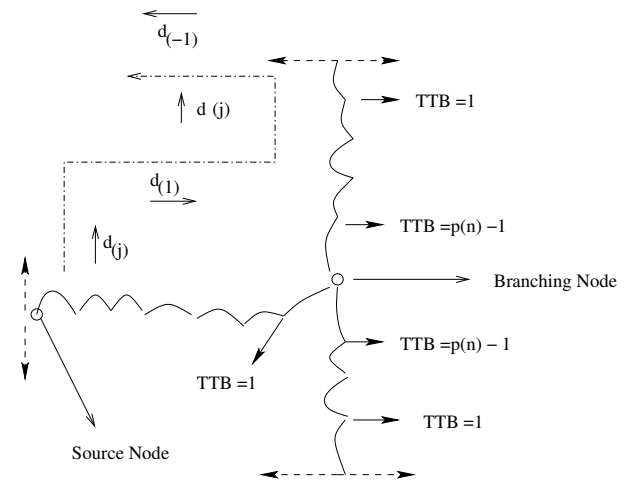

Fig. 7. Illustrates the query branching in sensor networks. Note that the branches do not follow straight lines due to approximate direction knowledge.

an optimal number of transmissions, if an advertising radius of $A(n)=M(n)^{\alpha}, \alpha<\frac{1}{2}$ is allowed. We show this in the following theorem for rational angles.

Theorem 6.1: Consider any point $X=\left(1, \theta^{*}\right)$ on the boundary of a unit ball around the origin. Consider a branching query process as described above. Then, there exists a $0<b<$ $\infty$ such that $\forall<1 / 2$.

$$
\min _{Y \in \mathcal{S} \frac{b}{M(n)}}\|Y-X\|_{\mathcal{L}_{2}} \leq M(n)^{\alpha} \quad \text { (prob.) }
$$

Moreover,by iteration $\frac{b}{M(n)}$, the total number of transmissions

$$
T(n)=O\left(\frac{1}{M(n)}^{\gamma}\right), \forall \gamma>1
$$

Proof: We first show Theorem 6.1 for $\theta^{*} \in\left[0, \frac{\pi}{4}\right]$ such that $\tan \theta^{*}$ is rational. The result follows for any $\theta^{*} \in\left[0, \frac{\pi}{4}\right]$ by the density of rationals $\mathbb{Q}$ in $\mathbb{R}$ and by the continuity of $\tan \theta^{*}$ on $\left[0, \frac{\pi}{4}\right]$. For any other $\theta^{*} \notin\left[0, \frac{\pi}{4}\right]$, the result follows, by symmetry.

The main steps of the proof are as follows.

1) We employ a $p(n)=\frac{1}{M(n) \log \log \frac{1}{M(n)}}$ to create slowly branching trees,

2) We show the existence of a path in the binary tree with a mean angular drift along $\theta^{*}$.

3) We then show that the path lies within a radius $M(n)^{\alpha}, \quad \alpha<\frac{1}{2}$ about the destination $X$.

Firstly, we describe the construction of the path in the binary tree. Let $\tan \theta^{*}=\frac{r}{q}$. Recall that the branching occurs exactly once every $p(n)=\frac{1}{M(n) \log \log \frac{1}{M(n)}}$ hops in each query. Further, note that at each branching, exactly two queries are sent along the two perpendicular directions to the original direction along which the query was traveling. That is, if a query traveling along direction $d_{(1)}$ branched, the two new queries would be directed along $d_{(j)}$ and $d_{(-j)}$. Consider the initial queries sent along the direction $d_{(j)}$ and $d_{(-j)}$ by the source node $S_{0}$.

1) We denote by $\mathfrak{D}_{1}:=\left(d_{(j)} ; d_{(1)} ; d_{(j)} ; d_{(-1)}\right)$, a sequence of the directions of branchings followed by the query, as depicted in Figure 7(in dotted lines). In particular $\left(d_{(j)} ; d_{(1)} ; d_{(j)} ; d_{(-1)}\right)$ defines the path of a query through four successive branchings; the direction followed at each branching provided by the sequence of directions. Similarly, we also define another sequence of branchings $\mathfrak{D}_{2}:=\left(d_{(-j)} ; d_{(1)} ; d_{(j)} ; d_{(1)}\right)$. From the construction of the tree, the expected position of the query, after the branchings $\left(d_{(j)} ; d_{(1)} ; d_{(j)} ; d_{(-1)}\right)$ is $c p(n) M(n)\left(2 d_{(j)}\right)$. The expected position of the query after the sequence of branchings $\left(d_{(-j)} ; d_{(1)} ; d_{(j)} ; d_{(1)}\right)$ is given by $c p(n) M(n)\left(2 d_{(1)}\right)$.

2) Consider the sequence of branchings obtained by following $r$ branchings of type $\mathfrak{D}_{1}$, followed by $q$ branchings of type $\mathfrak{D}_{2}$, i.e., the sequence $\mathfrak{D}_{r q}=$ $(\underbrace{\mathfrak{D}_{1} ; \ldots ; \mathfrak{D}_{1}}_{r \text { terms }} ; \underbrace{\mathfrak{D}_{2} ; \ldots ; \mathfrak{D}_{2}}_{q \text { terms }})$. The expected position of the query after the sequence of branchings $\mathfrak{D}_{r q}$ is $c p(n) M(n)\left(2 r d_{(1)}+2 q d_{(j)}\right)$.

3) We construct the sequence of branchings formed by following $l^{*}$ branchings of type branchings $\mathfrak{D}_{r q}$, where $l^{*}=\frac{1}{\sqrt{r^{2}+q^{2}} c p(n) M(n)}$. That is, $\mathfrak{D}_{\theta *}=(\underbrace{\mathfrak{D}_{r q} ; \ldots ; \mathfrak{D}_{r q}}_{l^{*} \text { terms }})$. Note that the expected position of the query after the sequence of branchings $\mathfrak{D}_{\theta *}$ is

$l^{*} \times c p(n) M(n)\left(2 r d_{(1)}+2 q d_{(j)}\right)=\left(d_{(1)} \cos \theta *+d_{(j)} \sin \theta *\right)$ $=(1, \theta *)($ in polar coordinates. $)$

In effect, we construct a path with mean drift along $\theta^{*}$, by appending a series of branchings. Note that the number of iterations to reach the end of the sequence $D_{\theta^{*}}$ is $l^{*} \times(r+$ $q) \times 4 \times p(n)=\frac{b}{M(n)}$. Thus, by construction, we show the existence of a path such that the mean position after $b$ iterations is the destination node $X$. We now show that the position of the path after the sequence of branchings $\mathfrak{D}_{\theta *}$ is within a distance $M(n)^{\alpha}$ of its mean $X=(1, \theta *)$, for all $\alpha<.5$, with high probability.

Now, let $\Gamma$ correspond to a binary tree created by a query along the direction $d_{(j)}$ from the source node. Notice that the position of the path $D_{\theta^{*}}$ is an element of this tree, at depth $b$. We denote position of the query after the sequence $D_{\theta^{*}}$ by the random variable $Z_{t}^{b}$, where $t \in\left\{1, \cdots, 2^{b-1}-1\right\}$. Thus, the position of the query is given by (depth $b$, leaf $t$ )

$$
Z_{t}^{b}=\sum_{i=0}^{b-1} Y_{\left[\frac{t}{2^{i}}\right]}^{b-i}
$$

Let $L_{1}=\left\{i: E\left(Y_{\left[\frac{t}{2^{i}}\right]}^{b-i}\right)=d_{(1)}\right\}$, that is, the set of indices such that the query is along direction $d_{(1)}$. Similarly, we define $L_{2}=\left\{i: E\left(Y_{\left[\frac{t}{2^{i}}\right]}^{b-i}\right)=d_{(j)}\right\}, L_{3}=\left\{i: E\left(Y_{\left[\frac{t}{2^{i}}\right]}^{b-i}\right)=d_{(-1)}\right\}$ and $L_{4}=\left\{i: E\left(Y_{\left[\frac{t}{2^{i}}\right]}^{b-i}\right)=d_{(-j)}\right\}$.

Since these sets are constructed deterministically, we rewrite sum in (34) as follows.

$$
Z_{t}^{b}=\sum_{i \in L_{1}} Y_{\left[\frac{t}{2^{i}}\right]}^{b-i}+\sum_{i \in L_{2}} Y_{\left[\frac{t}{2^{i}}\right]}^{b-i}+\sum_{i \in L_{3}} Y_{\left[\frac{t}{2^{i}}\right]}^{b-i}+\sum_{i \in L_{4}} Y_{\left[\frac{t}{2^{i}}\right]}^{b-i}
$$

Notice that $Y_{\left[\frac{t}{2^{i}}\right]}^{b-i}$ for $i \in\left\{L_{r}, r=1\right.$ to 4$\}$ are i.i.d. random variables. Fox example, $Y_{\left[\frac{t}{2}\right]}^{b-i}, i \in L_{1}$ is a random variable corresponding to a query along the direction $d_{(1)}$. Thus, each random variable in this set is a sum of $p(n)$ hops along direction $d_{(1)}$. Thus,

$$
Y_{\left[\frac{t}{2^{i}}\right]}^{b-i}=d_{(1)}\left(\sum_{m=1}^{p(n)} R_{m}\right)
$$


where $R_{m}$ are i.i.d. random variables with support $[0, M(n)]$ and mean $c M(n)$. (See discussion in VI.A for the above construction). Since each random variable $Y_{\left[\frac{t}{2^{i}}\right]}^{b-i}$ is a sum of $p(n)$ random variables of kind $R_{m}$, we have the following claim.

Claim 1: Let . $5<\beta<1$. Then,

$\mathbb{P}\left(\left\|Y_{\left[\frac{t}{2^{i}}\right]}^{b-i}-c(M(n) p(n)) d_{(1)}\right\|>M(n)(p(n))^{\beta}\right) \leq e^{-p(n)^{2 \beta-1} \epsilon}$

for some $\epsilon>0$.

Proof: By construction,

$$
\begin{array}{r}
\mathbb{P}\left(\left\|Y_{\left[\frac{t}{2^{i}}\right]}^{b-i}-(M(n) p(n) c) d_{(1)}\right\|>M(n)(p(n))^{\beta}\right) \\
\leq \mathbb{P}\left(\sum_{m=1}^{p(n)}\left(R_{m}-M(n) c\right)>M(n)(p(n))^{\beta}\right) .
\end{array}
$$

Let $\tilde{R}_{m}=\frac{1}{M(n)} R_{m}$. Then, note that

$$
\begin{array}{r}
\mathbb{P}\left(\sum_{m=1}^{p(n)}\left(R_{m}-M(n) c\right)>M(n)(p(n))^{\beta}\right) \\
=\mathbb{P}\left(\sum_{m=1}^{p(n)}\left(\tilde{R}_{m}-c\right)>(p(n))^{\beta}\right) \\
=\mathbb{P}\left(\frac{1}{(p(n))^{\beta}} \sum_{m=1}^{p(n)}\left(\tilde{R}_{m}-c\right)>1\right) \\
\leq e^{-p(n)^{2 \beta-1} \epsilon}, \epsilon>0 .
\end{array}
$$

A similar inequality can be derived for the negative side as well. We skip the details for brevity. The inequality in (39) follows from the result ([3]) in moderate deviations about the mean, for sums of random variables.

Consider the path $\mathfrak{D}_{\theta *}$. It is easily seen that there are $(2 q+$ $r) * l^{*}$ queries in the path along direction $d_{(1)},(2 r+q) * l^{*}$ in the path along direction $d_{(j)},(r) * l^{*}$ in the path along direction $d_{(-1)}$ and $(q) * l^{*}$ in the path along direction $d_{(-j)}$. Note that this implies that for the first term on the R.H.S of (35)

$$
\begin{aligned}
\mathbb{P}\left(\left(\left\|\sum_{i \in L_{1}} Y_{\left[\frac{t}{2^{i}}\right]}^{b-i}-\frac{2 q+r}{\sqrt{r^{2}+q^{2}}} d_{(1)}\right\|\right.\right. & \left.>K(p(n))^{\beta-1}\right) \\
& \leq e^{-p(n)^{2 \beta-1} \epsilon_{1}},
\end{aligned}
$$

for some $\epsilon_{1}>0$, and $K<\infty$. Using a similar bound for all the terms on the R.H.S of (35), and noting that

$$
\begin{aligned}
X= & \frac{2 q+r}{\sqrt{r^{2}+q^{2}}} d_{(1)}+\frac{2 r+q}{\sqrt{r^{2}+q^{2}}} d_{(j)}+ \\
& \frac{r}{\sqrt{r^{2}+q^{2}}} d_{(-1)}+\frac{q}{\sqrt{r^{2}+q^{2}}} d_{(-j)},
\end{aligned}
$$

we find that

$$
\mathbb{P}\left(\left\|Z_{t}^{b}-X\right\|>K_{1}(p(n))^{\beta-1}\right) \leq e^{-p(n)^{2 \beta-1} \epsilon_{2}}
$$

for some $\epsilon_{2}>0$, and $K_{1}<\infty$. Since $p(n)=$ $\frac{1}{M(n) \log \log \frac{1}{M(n)}}$, the quantity $K(p(n))^{\beta-1}=O\left(M(n)^{\alpha}\right)$ for all $\alpha<1-\beta$, and thus, (32) follows.

The total number of transmissions in any binary tree by iteration $\frac{b}{M(n)}$ is given by $p(n) \times 2^{K \log \log \frac{1}{M(n)}}$, where $K \log \log \frac{1}{M(n)}$ is the depth of the binary tree. Notice that

\begin{tabular}{|c|c|c|c|c|}
\hline M(n) & \multicolumn{2}{|c|}{ Sub-Critical } & \multicolumn{2}{c|}{ Super-Critical } \\
\hline & Parameter & Prob. & Parameter & Prob. \\
\hline 0.11 & $\mathrm{c}=1.4$ & 0.12 & $\mathrm{c}=2.0$ & 0.99 \\
0.09 & $\mathrm{c}=1.4$ & 0.09 & $\mathrm{c}=1.9$ & 0.93 \\
0.07 & $\mathrm{c}=1.4$ & 0.02 & $\mathrm{c}=2.0$ & 0.99 \\
\hline
\end{tabular}

TABLE II

ZERo INFORMATION - SUCCESS PROBABILITY WITH 15/M(n) ITERATIONS

\begin{tabular}{|c|c|c|c|c|}
\hline M(n) & \multicolumn{2}{|c|}{ Sub-Critical } & \multicolumn{2}{c|}{ Super-Critical } \\
\hline & Parameter & Prob. & Parameter & Prob. \\
\hline 0.11 & $\gamma=1.5$ & 0.25 & $\gamma=3.0$ & 1.00 \\
0.09 & $\gamma=1.5$ & 0.2 & $\gamma=2.7$ & 0.97 \\
0.07 & $\gamma=1.5$ & 0.07 & $\gamma=2.7$ & 0.97 \\
\hline
\end{tabular}

TABLE III

Source Quadrant Information - Success Probability with $2 / M(n)$ ITERATIONS

we create four binary trees, and hence the total number of transmissions $T(n)=4 p(n) * \log \frac{1}{M(n)}^{K}$, which is order-wise smaller than $\frac{1}{M(n)}^{\gamma}$ for all $\gamma>1$.

Remark 6.1: Thus, the results in this section show that even with approximate local direction information, the number of transmissions to reach an advertisement region of $\sqrt{M(n)}$ is only $\Theta\left(\frac{1}{M(n)}^{\gamma}\right)$. That is, a polynomial number of transmissions are sufficient to spread queries efficiently to lattice points in networks with approximate local direction.

\section{Simulation Results}

In this section, we provide simulation results for the strategies considered in this paper. In all the simulations, we set the source location to be at $(0,0)$. For the first three broadcast strategies, the destination is chosen to be at $(1,0)$. For spatial sampling (broadcasting on a lattice), we choose the destination to be at $(.7, .7)$ (for better representation). For each of the strategies, we provide simulation results to show the probability of "success" (appropriately defined for each

\begin{tabular}{|c|c|c|c|c|}
\hline M(n) & \multicolumn{2}{|c|}{ Sub-Critical } & \multicolumn{2}{c|}{ Super-Critical } \\
\hline & Parameter & Prob. & Parameter & Prob. \\
\hline 0.11 & $C=1$ & 0.26 & $C=2.0$ & 0.99 \\
0.09 & $C=1$ & 0.25 & $C=2.0$ & 1.00 \\
0.07 & $C=1$ & 0.12 & $C=2.0$ & 1.00 \\
\hline
\end{tabular}

TABLE IV

STATE INFORMATION - SUCCESS PROBABILITY With 5/M( $n)$ ITERATIONS

\begin{tabular}{|c|c|c|c|c|}
\hline M(n) & \multicolumn{2}{|c|}{ Sub-Critical } & \multicolumn{2}{c|}{ Super-Critical } \\
\hline & Parameter & Prob. & Parameter & Prob. \\
\hline 0.06 & $\alpha=0.8$ & 0.18 & $\alpha \mathrm{c}=0.4$ & 0.94 \\
0.04 & $\alpha=0.8$ & 0.16 & $\alpha \mathrm{c}=0.4$ & 0.90 \\
0.02 & $\alpha=0.8$ & 0.20 & $\alpha \mathrm{c}=0.4$ & 0.98 \\
\hline
\end{tabular}

TABLE V

Spatial SAMPLING By BRANCHING - SUCCess PRobability With $15 / M(n)$ ITERATIONS 


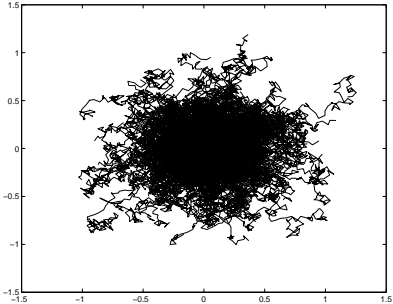

(a) Zero Information

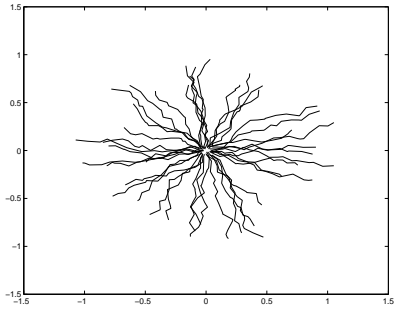

(b) Source Quadrant Information

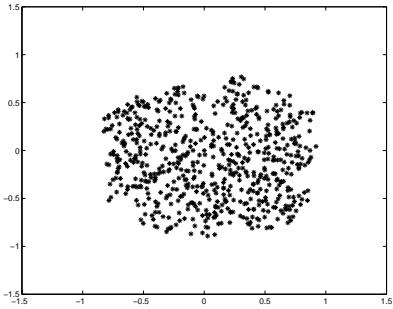

(c) State Information

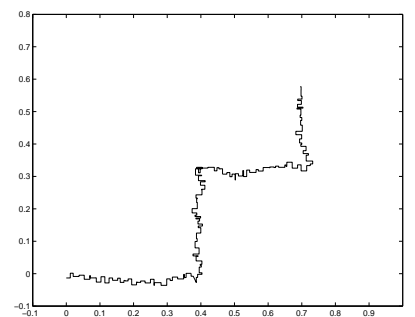

(d) Spatial Sampling - Local Direction Information

Fig. 8. Sample Paths of Broadcasts in Networks with Local Information

strategy) for varying parameters and averaged over 50 runs. The transmission radius is chosen such that the number of hops between the source and destination is about $10-15$.

In Table II, we have provided the probability that a query reaches within an $M(n)$ distance of the destination (success) within $\Theta(1 / M(n))$ for the case where there is no information. We have earlier shown that an exponential number of queries are necessary and sufficient for broadcasting without information. To illustrate this by simulation, we have chosen two constants $c_{i}, i=1,2$ and the number of parallel queries sent by the source is $c_{i}^{1 / M(n)}$. The table shows that if $c_{1}$ is chosen small enough (but still resulting in an exponential number of queries), the probability of success is small, while a larger value of $c_{2}$ results in a success probability that is close to '1', as predicted in Section III. A sample path of the parallel query strategy is illustrated in Figure 8.

In Table III, nodes have source-quadrant information, thus requiring only a polynomial number of parallel queries (with the exponent being 2.5). In the table we have chosen two growth exponents $\gamma_{1}<2.5<\gamma_{2}$ (i.e, the number of parallel queries is $\left.\left(1 / M(n)^{\gamma}\right)\right)$, and the results demonstrate a "subcritical" rate and a "super-critical" rate (i.e., the probabilities are close to '0' or '1' respectively). In Table IV, a similar result has been plotted for the suppression based strategy (local state-information), with up to $C \log (1 / M(n))$ new transmitters chosen (prior to suppression). Again, we can see the subcritical and super-critical behavior. Finally, in Table V, we have shown a sub-critical and super-critical behavior for lattice flooding, with an advertisement radius $A(n)=M(n)^{\alpha}$. We have chosen $\alpha_{1}<0.5<\alpha_{2}$ to show that the advertisement region needs to be large enough for success. Sample paths of all the strategies described above are illustrated in Figure 8.

\section{REFERENCES}

[1] D. Braginsky and D. Estrin. Rumor routing algorithm for sensor networks. In First Workshop on Sensor Networks and Applications (WSNA), September 2002.

[2] J. Cartigny, D. Simplot, and I. Stojmenovic. Localized minimum-energy broadcasting in ad-hoc networks. In IEEE Trans. Parallel Distrib. Syst. 13(1), pages 14-25, 2002.

[3] C. Chang, D. Yao, and T. Zajic. Large deviations, moderate deviations, and queues with long-range dependent inputs. Adv. Appl. Prob., 31:254277, 999.

[4] M. Chang, N.and Liu. Optimal controlled flooding search in a large wireless network. In Proc. 3rd International Symposium on Modeling and Optimization in Mobile, Ad Hoc and Wireless Networks, 2005.

[5] M. Chu, H. Haussecker, and F. Zhao. Scalable information-driven sensor querying and routing for ad hoc heterogeneous sensor networks. Technical Report P2001-10113, Xerox PARC, 2001.

[6] A. El Gamal, J. Mammen, B. Prabhakar, and D. Shah. Throughput delay trade-off in wireless networks. In Proceedings of IEEE Infocom, Hong Kong, March 2004.
[7] W. Feller. An Introduction to Probability Theory and Its Applications, Volume II. Wiley, 1966.

[8] P. Gupta and P. R. Kumar. Critical power for asymptotic connectivity in wireless networks. In Stochastic Analysis, Control, Optimization and Applications: A Volume in Honor of W.H. Fleming. Edited by W.M. McEneany, G. Yin, and Q. Zhang, pages 547-566, Boston, 1998. Birkhauser.

[9] Z.J. Haas, J.Y. Halpern, and L Li. Gossip-based ad hoc routing. In Proceedings ofIEEE INFOCOM, 2002.

[10] B. Hajek. Minimum mean hitting times of Brownian motion with constrained drift. In Proceedings of the 27th Conference on Stochastic Processes and Their Applications, July 2000.

[11] C. Ho, K. Obraczka, G. Tsudik, and K. Viswanath. Flooding for reliable multicast in multi-hop ad hoc networks. In Proceedings of DIALM, pages 64-71, 1999.

[12] C. Intanagonwiwat, R. Govindan, and D. Estrin. Directed diffusion: A scalable and robust communication paradigm for sensor networks. In Proceedings of ACM Mobicom, Boston, MA, August 2000.

[13] S.Y. Ni, Y.C. Tseng, Y.S. Chen, and Sheu J.P. The broadcast storm problem in a mobile ad hoc network. In Proceedings of the ACM/IEEE International Conference on Mobile Computing and Networking (MOBICOM), pages 151-162, 1999.

[14] L. Orecchia, A. Panconesi, A. Petrioli, and A. Vitaletti. Localized techniques for broadcasting in wireless sensor networks. In Proceedings of the 2004 joint workshop on Foundations of mobile computing, pages 41-51, 2004.

[15] Wei Peng and Xi-Cheng Lu. On the reduction of broadcast redundancy in mobile ad hoc networks. In Proc. First Ann.Workshop Mobile and Ad Hoc Networking and Computing, pages 129-130, Apr. 2000.

[16] N. Sadagopan, B. Krishnamachari, and A. Helmy. The ACQUIRE mechanism for efficient querying in sensor networks. In IEEE International Workshop on Sensor Network Protocols and Applications (SNPA'03), May 2003.

[17] S. Shakkottai. Asymptotics of query strategies over a sensor network. IEEE Transactions on Automatic Control, 50(5):594 - 606, May 2005.

[18] I. Stojmenovic and M. Seddigh. Broadcasting algorithms in wireless networks. In Proceedings of the International Conference on Advances in Infrastructure for Electronic Business, Science, and Education on the Internet SSGRR, 2000.

[19] I. Stojmenovic, M. Seddigh, and J.D. Zunic. Dominating sets and neighbor elimination-based broadcasting algorithms in wireless networks. In IEEE Trans. Parallel Distrib. Syst. 13(1), pages 14-25, 2002.

[20] S. Subramanian and S. Shakkottai. Geographic Routing with Limited Information in Sensor Networks. In Proceedings of Information Processing in Sensor Networks, pages 269-276, April 2005.

[21] S. Subramanian, S. Shakkottai, and A. Arapostathis. Broadcasting in Sensor Networks: The Role of Local Information. WNCG Technical Report, University of Texas at Austin, January 2006.

[22] J.E Wieselthier, G.D. Nguyen, and A. Ephremides. On the construction of energy-efficient broadcast and multicast trees in wireless networks. In Proceedings of IEEE INFOCOM, pages 589-594, Apr. 2000.

[23] B. Willams and T. Camp. Comparison of broadcasting techniques for mobile ad hoc networks. In Proceedings of the 3rd ACM international symposium on Mobile ad hoc networking and computing, 2002. 